\title{
Cyclopeptide toxins of lethal amanitas: compositions, distribution and phylogenetic implication
}

\author{
Shanshan Tang ${ }^{\text {a, }}$, Qian Zhou ${ }^{\text {a, }}$, Zhengmi He ${ }^{\text {a }}$, Tao Luo ${ }^{a}$, Ping Zhang ${ }^{\text {a }}$, Qing Cai ${ }^{\text {b }}$, Zhuliang Yang ${ }^{\text {b }}$ \\ Jia Chen ${ }^{\mathrm{c}}$, Zuohong Chen ${ }^{\text {a, * }}$ \\ ${ }^{a}$ College of Life Science, Hunan Normal University, Changsha 410081, China. \\ ${ }^{\mathrm{b}}$ Key Laboratory for Plant Diversity and Biogeography of East Asia, Kunming Institute of Botany, \\ Chinese Academy of Sciences, Kunming, Yunnan 650201, China \\ ${ }^{c}$ State Key Laboratory of Toxicology and Medical Countermeasures, and Laboratory of Toxicant \\ Analysis, Institute of Pharmacology and Toxicology, Academy of Military Medical Sciences, \\ Beijing, 100850, China \\ * Corresponding author: Zuo-Hong Chen, College of Life Science, Hunan Normal University, 36 \\ Lushan Road, Changsha 410081, China. \\ Tel.: +86 73188872557 ; Fax: +86 73188883310 \\ E-mail address: chenzuohong@263.net \\ $\S$ Shanshan Tang and Qian Zhou contributed equally to this work.
}

\begin{abstract}
Lethal amanitas (Amanita sect. Phalloideae) are responsible for $90 \%$ of all fatal mushroom poisonings. Since 2000, more than ten new lethal Amanita species have been discovered and some of them had caused severe mushroom poisonings in China. However, the contents and distribution of cyclopeptides in these lethal mushrooms remain poorly known. In this study, the diversity of major cyclopeptide toxins in seven Amanita species from Eastern Asia and three species from Europe and North America were systematically analyzed, and a new approach to inferring
\end{abstract}


phylogenetic relationships using cyclopeptide profile was evaluated for the first time. The results showed that there were diversities of the cyclopeptides among lethal Amanita species, and cyclopeptides from A. rimosa and A. fuligineoides were reported for the first time. The amounts of amatoxins in East Asian Amanita species were significantly higher than those in European and North American species. The analysis of distribution of amatoxins and phallotoxins in various Amanita species demonstrated that the content of phallotoxins was higher than that of amatoxins in $A$. phalloides and A. virosa. In contrast, the content of phallotoxins was significantly lower than that of amatoxins in all East Asian lethal Amanita species tested. However, the distribution of amatoxins and phallotoxins in different tissues showed the same tendency. Eight cyclopeptides and three unknown compounds were identified using cyclopeptide standards and high-resolution MS. Based on the cyclopeptide profiles, phylogenetic relationships of lethal amanitas were inferred through a dendrogram generated by UPGMA method. The results showed high similarity to the phylogeny established previously based on the multi-locus DNA sequences.

Keywords: Amanita, Amatoxins, Phallotoxins, Gene family, Phylogeny, HPLC

\section{Highlights}

- The major cyclopeptides in lethal amanitas were identified using cyclopeptide standards and high-resolution MS method.

- The composition and distribution of amatoxins and phallotoxins of the lethal amanitas from Eastern Asia, Europe and North America were compared.

- The dendrogram based on cyclopeptide chromatographic profiles was similar to the phylogenetic tree based on ITS and multi-locus DNA sequences. 


\section{Introduction}

The genus Amanita is a cosmopolitan genus comprising about 600 described species, which occur worldwide (Yang et al., 2016). This genus is important to humans as it contains many deadly poisonous species. The poisoning by Amanita mushrooms can be classified into three categories: neurotoxicity, nephrotoxicity and hepatotoxicity according to the syndromic classification (Diaz, 2005). Neurotoxic Amanita species such as A. muscaria, A. pantherina contain ibotenic acid and muscimol, which cause hallucinogenic effects (Benjamin, 1992; Michelot and Melendez-Howell, 2003). Nephrotoxic Amanita species such as A. smithiana, A. proxima, and A. pseudoporphyria can induce acute renal failure, the responsible toxin for which is likely to be 2-amino-4,5-hexadienoic acid (Saviuc \& Danel, 2006; Kirchmair et al., 2012). Hepatotoxic Amanita species, such as A. phalloides, A. verna and A. virosa in Europe and North America, and A. exitialis, A. fuliginea and A. subjunquillea in East Asia, contain cyclopeptides and are responsible for the liver failure and death of humans. These cyclopeptide-containing Amanita species account for over $90 \%$ of all fatal mushroom poisonings worldwide (Karlson-Stiber and Persson, 2003; Berger and Guss, 2005; Chen et al., 2014).

The lethal amanitas are a group of cyclopeptide-containing mushrooms of Amanita sect. Phalloideae, comprising ca. 37 described species worldwide with majority of them distributed in the Northern Hemisphere (Cai et al., 2014). In Europe and North America, the lethal Amanita species include A. phalloides, A. verna, A. virosa, A. ocreata, A. bisporigera, A. suballiacea, A. tenuifolia, and A. phalloides var. alba, which have caused human deaths (Enjalbert et al., 2002; Karlson-Stiber and Persson, 2003; Kaya, et al., 2013a). Some of these European and North American poisonous Amanita species, such as A. phalloides and A. verna, were also reported from East Asia (Imazeki et al., 1988; 
Mao, 2006). Recent comprehensive taxonomic studies demonstrated that these earlier identifications were incorrect (Yang, 2000, 2005). On the other hand, many new lethal Amanita taxa, endemic to East Asia, such as A. subjunquillea S. Imai (Imai, 1933), A. fuliginea Hongo (Hongo, 1953), A. subjunquillea var. alba Zhu L. Yang (Yang, 1997), A. exitialis Zhu L. Yang \& T. H. Li (Yang and Li, 2001), A. fuligineoides P. Zhang \& Zhu L. Yang, A. rimosa P. Zhang \& Zhu L. Yang, A. pallidorosea P. Zhang \& Zhu L. Yang (Zhang et al., 2010) and A. subpallidorosea Hai J. Li (Li et al,. 2015) have been described from China and Japan. Recently, 28 phylogenetic species were recognized by multi-locus phylogenetic analyses assisted with morphological studies, 14 of which represented putatively new species (Cai et al., 2014). Many of these Eastern Asian lethal Amanita species have caused serious mushroom poisonings in China (Yang and Li, 2001; Chen et al., 2014; Cao et al., 2011; Li et al., 2015).

Up to now, ca. twenty-two cyclopeptide toxins from Amanita species have been identified, they are classified into three major groups: amatoxins (bicyclic octapeptides), phallotoxins (bicyclic heptapeptides) and virotoxins (monocyclic heptapeptides) (Wieland, 1986; Li and Oberlies, 2005; Clarke et al., 2012). Amatoxins are responsible for fatal human poisonings through inhibiting RNA polymerase II, subsequently blocking the synthesis of proteins, and eventually leading to cell death (Wieland, 1986). The detailed mode of action and the crystal structure of $\alpha$-amanitin have been demonstrated (Nguyen et al., 1996; Wang et al., 2006; Kaplan et al, 2008). Phallotoxins and virotoxins do not play a role in human poisonings for they are not absorbed by intestinal cells, although they can cause death of mice and rats within 1-2 h after interperitoneal administration (Karlson-Stiber \& Persson, 2003; Li and Oberlies, 2005). The phallotoxins specifically bind to F-actin, thus strongly stabilizing the structure of the assembled filaments (Wieland, 1986).

The contents and compositions of cyclopeptides in leathal amanitas are variable among different 
species. Amatoxins and phallotoxins were firstly isolated from A. phalloides, whereas virotoxins were first isolated in A. virosa (Faulstich et al., 1980; Wieland, 1986). In the early years, the quantities of amatoxins and phallotoxins in various cyclopeptide-containing mushrooms including A. phalloides, A. verna, A. virosa, A. ocreata, A. bisporigera, Galerina marginata, Lepiota brunneoincarnata were determined by various chromatographic methods such as column chromatography, thin-layer chromatography (TLC) or high-performance TLC (HPTLC), coupled with spectrophotometric or colorimetric method for the identification of toxic compounds (Wieland, 1986). Enjalbert et al. (1992) established a high performance liquid chromatographic (HPLC) method combined with UV absorbance, which allows simultaneous determination of up to eight amatoxins and phallotoxins. Later, HPLC method combined with UV absorbance or mass spectrometry was widely applied to various Amanita mushrooms, including European and North American species such as A. phalloides (Enjalbert et al., 1993, 1996, 1999; Sgambelluri et al., 2014; Kaya et al., 2015), A. phalloides var. alba (Kaya et al., 2013), A. bisporigera (Mcknight et al., 2010) and A. virosa (Ahmed et al., 2010; Sgambelluri et al., 2014), and the Eastern Asian species such as A. exitialis (Hu et al., 2012), A. subjunquillea (Bao et al., 2005), A. fuliginea (Chen et al., 2003) and A. pallidorosea (Wang et al., 2011). However, most of these studies focused on a small subset of the cyclopeptides, mainly $\alpha$-, $\beta$-amanitin and/or phalloidin. Furthermore, the toxin profiles obtained in different laboratories lacked comparability, largely due to differences among extraction methods of toxins and the conditions of HPLC. In addition, the cyclopeptide toxins of the lethal A. fuligineoides and A. rimosa mushrooms remain unknown.

The objectives of this study are: (i) to analyze the composition and distribution of major cyclopeptide toxins of the lethal amanitas from Eastern Asia, Europe and North America by means of HPLC, (ii) to identify cyclopeptides in lethal amanitas by mass spectrometry, and (iii) to assess their 
phylogenetic relationships based on the cyclopeptide profiles.

\section{Materials and methods}

2. 1. Mushroom collection and sample conditions

Twenty-one Amanita samples collected from East Asia, Europe and North America were included in this study. The samples determined in this study were deposited in Mycological Herbarium of Hunan Normal University (MHHNU), the Cryptogamic Herbarium of Kunming Institute of Botany, Chinese Academy of Sciences (HKAS) and Mycological Herbarium of Guangdong Institute of Microbiology (GDGM). Information on the collections and their ITS accession number in GenBank are listed in Table 1 and Fig. 1. According to the sample conditions, the tissues used for toxin composition study varied. When available, fully developed, complete Amanita carpophores were used, except for $A$. bisporigera, A. phalloides, A. virosa, A. fuligineoides and A. subjunquillea, in which cases only small sections of pileus were available. For analysis of toxin composition in different tissues, the carpophores of A. exitialis, A. fuliginea, A. pallidorosea and A. rimosa were divided into three parts: pileus, stipe and volva.

\section{2 Mushroom Identification and phylogenetic analysis based on ITS sequences}

All Amanita species were identified by both morphological and molecular phylogenetic evidence. DNA extraction, and PCR amplification, sequencing and alignment of ITS sequences followed Zhang et al. (2010) and Cai et al. (2014). The phylogenetic tree was generated with both maximum likelihood (ML) (Felsenstein, 1981) and Bayesian methods (Ronquist and Huelsenbeck 2003) with the parameters employed in Cai et al. (2014), except that the runs of Bayesian inference were performed for 10 million generations. 
Table 1. List of samples analyzed in this study.

\begin{tabular}{|c|c|c|c|c|}
\hline Scientific Name & Species no. & Geographic origin & Collection time & $\begin{array}{l}\text { ITS GenBank } \\
\text { accession No. }\end{array}$ \\
\hline A. bisporigera G. F. Atk. & MHHNU7224 & Hamilton, Canada & 2009. 8.26 & KU311692 \\
\hline A. exitialis Zhu L. Yang \& T. H. Li & MHHNU30297 & Guangzhou, Guangdong & 2009. 3.21 & KT003192 \\
\hline A. exitialis & MHHNU30298 & Guangzhou, Guangdong & 2009.3.22 & KR996716 \\
\hline A. exitialis & MHHNU 30937 & Shenzhen, Guangdong & 2014.3.26 & KR996717 \\
\hline A. fuliginea Hongo & MHHNU 30300 & Xingtan, Hunan & 2009. 6.10 & KT003190 \\
\hline A. fuliginea & MHHNU 30207 & Ningxiang, Hunan & 2003.6 .30 & KR996718 \\
\hline A. fuliginea ZP-1453 & MHHNU8953 & Youxian, Hunan & 2014.6.11 & KR870315 \\
\hline A. fuligineoides P. Zhang \& Zhu L. Yang & HKAS 52316 & Mangshan, Hunan & 2007.9.6 & FJ176721 \\
\hline A. oberwinklerana Zhu L. Yang \& Yoshim. Doi & MHHNU 7726 & Hupingshan, Hunan & 2012.9 .8 & KU311694 \\
\hline A. oberwinklerana & MHHNU 30819 & Guanzhou, Guangdong & 2013.5.28 & KT003191 \\
\hline A. pallidorosea P. Zhang \& Zhu L. Yang & MHHNU 8112 & Yanhe, Guizhou & 2014.7.18 & KU311697 \\
\hline A. pallidorosea & MHHNU 7354 & Hupingshan, Hunan & 2010.8 .31 & KU311693 \\
\hline A. phalloides (Fr.) Link. & HKAS 31457 & Tubingen,Germany & 1994. 9.10 & KT239525 \\
\hline A. phalloides & GDGM41101 & Lazio, Rome, Italy & 2014.10.6 & KT003193 \\
\hline A. rimosa P. Zhang \& Zhu L. Yang & MHHNU 8041 & Ningxiang, Hunan & 2014.6.27 & KU311696 \\
\hline A. rimosa & MHHNU 7954 & Youxian, Hunan & 2014.6.11 & KU311695 \\
\hline A. subjunquillea S. Imai & MHHNU 7049 & Shennongjia, Hubei & 2006.7.29 & FJ176731 \\
\hline A. subjunquillea & MHHNU 7751 & Hupingshan, Hunan & 2012.9 .10 & KR996715 \\
\hline A. virosa $(\mathrm{Fr}$.) Bertillon & HKAS 84859 & Germany & 2009. 9.20 & KR862367 \\
\hline A. virosa & HKAS56694 & Finland & 2009.10 .16 & JX998030 \\
\hline
\end{tabular}

\section{2. Extraction of the cyclopeptide toxins}

All samples were dried to constant weight and then ground by hand with a mortar and pestle. For each sample, $0.05 \mathrm{~g}$ ground materials was weighed, dispensed accurately into individual test tube, and extracted with $1 \mathrm{~mL}$ of $50 \%$ methanol (prepared with doubly distilled water) at $25{ }^{\circ} \mathrm{C}$. The test tubes were placed in a rotary shaker with a radius of $13 \mathrm{~mm}$ and shaken at $150 \mathrm{rpm}$ for $12 \mathrm{~h}$. Following 
centrifugation at $4000 \times \mathrm{g}$ for $15 \mathrm{~min}$, the supernatant was decanted and retained while the residue was resuspended in $1 \mathrm{~mL}$ of $50 \%$ methanol and extracted again as described above. The two supernatants were combined, freeze-dried, dissolved in $1 \mathrm{~mL}$ of doubly distilled water, and filtered through a micropore filter membrane $(0.22 \mu \mathrm{m})$ for HPLC analysis. The extraction was done in triplicate for each sample and the mean value was determined.
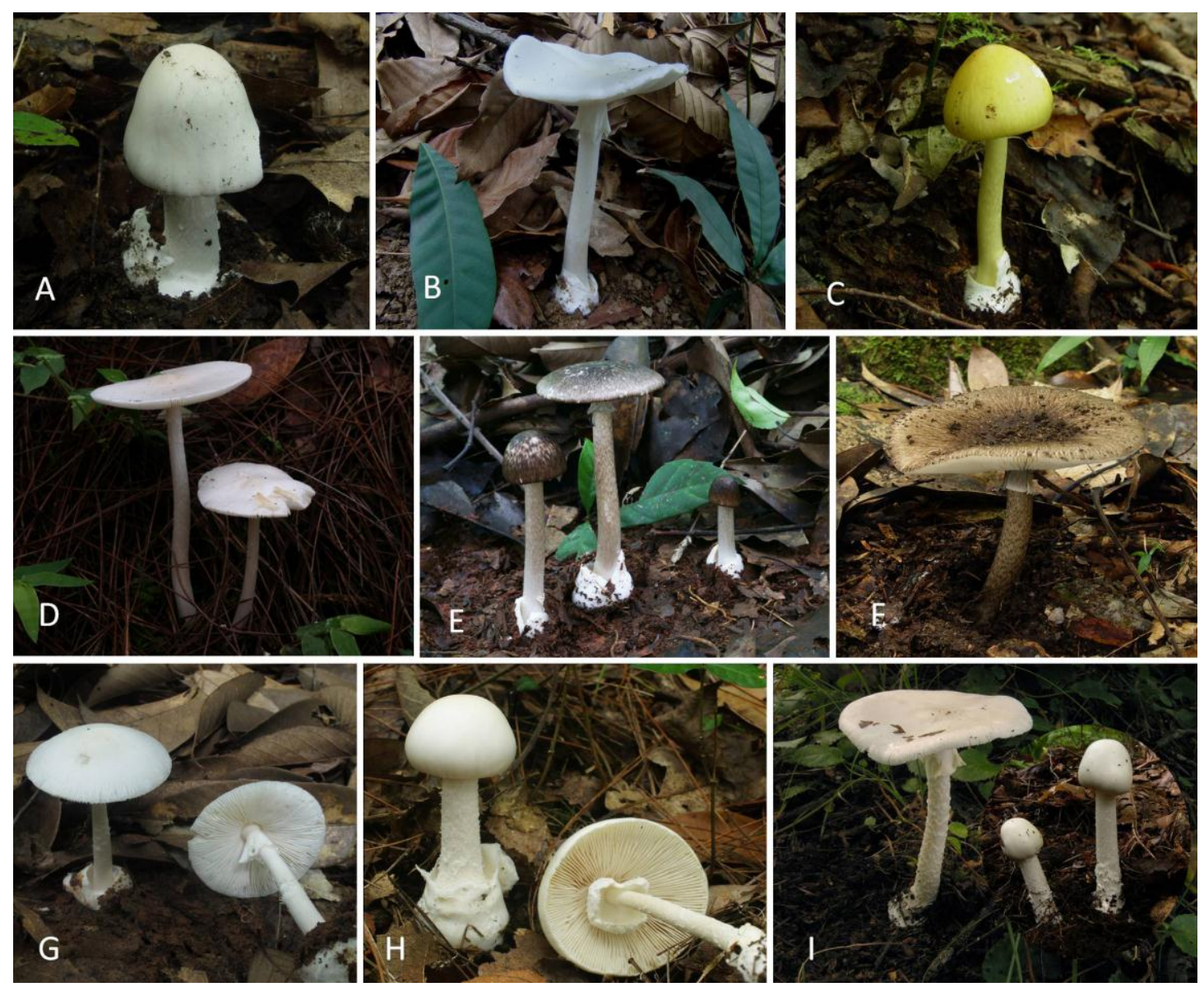

Fig. 1. Basidiomata of Amanita species $\quad$ A. A. bisporigera (MHHNU 7224); B. A. exitialis (MHHNU

30297); C. A. subjunquillea (MHHNU 7049); D. A. pallidorosea (MHHNU 8112); E. A. fuliginea

(MHHNU 30300); F. A. fuligineoides (HKAS 52316); G. A. rimosa (MHHNU 8041); H. A. oberwinklerana (MHHNU 7726); I. A. virosa (photos A, C, F, G, H by Ping Zhang; photos B, D, E by Zuohong Chen; photo I by Zhu L. Yang). 


\section{3. Standard samples and linear regression equation}

Standard samples of $\alpha$-amanitin ( $\alpha$-AMA, purchased from Merck-Calbiochem, Merck KGaA, Darmstadt, Germany), $\beta$-amanitin ( $\beta$-AMA, Sigma-Aldrich, St. Louis, MO USA), phallisacin (PSC, Sigma-Aldrich), phallacidin (PCD, Sigma-Aldrich) and phalloidin (PHD, Sigma-Aldrich) were dissolved in doubly distilled water to a concentration of $0.1 \mathrm{mg} \mathrm{mL}^{-1}$. A five-point curve was obtained with injections of $5,10,15,20$ and $30 \mu \mathrm{L}$ and the linear regression equation and correlation coefficient were calculated. The correlation regression coefficients of each curves were greater than 0.999 . $\alpha$-AMA, $\beta$-AMA, PSC, PCD and PHD in the samples were identified by comparison of retention times and co-injection with standards. Concentrations were calculated using peak areas of reference compounds.

\section{4. HPLC analysis}

Cyclopeptides were analyzed by reverse phase HPLC using a Waters Delta 600 HPLC system (Waters Inc., Milford, Massachusetts, USA) coupled with a Waters 2487 UV detector. Separation was carried out on a Sinochrom ODS-BP reverse phase HPLC column $(250 \mathrm{~mm} \times 4.6 \mathrm{~mm}$, particle size 10 $\mu \mathrm{m}$; Danlian Elite Analytical Instrument, Dalian, China) at $40{ }^{\circ} \mathrm{C}$. The absorbance of the eluates was monitored at $295 \mathrm{~nm}$. The mobile phase and elution profile were analyzed as described by Enjalbert et al. (1992) and Zhang et al. (2005). The mobile phase comprised (A) $0.02 \mathrm{~mol} \mathrm{~L}^{-1}$ aqueous ammonium acetate/acetonitrile $(90: 10 \mathrm{v} / \mathrm{v})$ and (B) $0.02 \mathrm{~mol} \mathrm{~L}^{-1}$ aqueous ammonium acetate/acetonitrile $(76: 24$ $\mathrm{v} / \mathrm{v}$ ), both adjusted to $\mathrm{pH} 5$ with glacial acetic acid. All solutions were degassed by sonication prior to use. The elution profile was as follows: (1) 0-15 min, 100-95\% A, 0-5\% B; (2) 15-35 min, 95-20\% A, 5-80\% B, then held for $5 \mathrm{~min}$; (3) 40-45 min, 20-0\% A, 80-100\% B, then held for $5 \mathrm{~min}$; (4) 50-60 $\min , 0-100 \% \mathrm{~A}, 100-0 \% \mathrm{~B}$. The mobile phase flow rate was $1 \mathrm{~mL} \mathrm{~min}{ }^{-1}$ and the sample injection 
volume was $20 \mu \mathrm{L}$. The peak eluates were collected for MS analysis.

\section{5. Mass spectrometry analysis (LC-MS)}

Liquid chromatography method: Waters Acquity UPLC, Waters BEH C18 LC column, 2.1×100 mm, $1.7 \mu \mathrm{m}$; mobile phase: A, $0.02 \mathrm{~mol} \mathrm{~L}^{-1}$ aqueous ammonium acetate/acetonitrile (90:10 v/v); B, $0.02 \mathrm{~mol} \mathrm{~L}{ }^{-1}$ aqueous ammonium acetate/acetonitrile $(76: 24 \mathrm{v} / \mathrm{v})$, both adjusted to $\mathrm{pH} 5$ with acetic acid; gradient elution: 0-1min, $0 \% \mathrm{~B}, 1-14 \mathrm{~min}, 0-100 \% \mathrm{~B}, 14-17 \mathrm{~min}, 100 \% \mathrm{~B}, 17-17.1 \mathrm{~min}$, $100-0 \% \mathrm{~B}, 17.1-20 \mathrm{~min}, 0 \% \mathrm{~B}$; flow rate was $0.25 \mathrm{~mL} \mathrm{~min}^{-1}$; injection volume was $5 \mu \mathrm{L}$.

High resolution mass spectrometry method: Waters Xevo G2-QTOF MS/MS; instrument parameters: electrospray positive ionization mode, capillary voltage $3 \mathrm{kV}$, sample cone voltage $30 \mathrm{~V}$, extraction cone voltage $4 \mathrm{~V}$, desolvation temperature $300{ }^{\circ} \mathrm{C}$, source temperature $100{ }^{\circ} \mathrm{C}$, desolvation gas $600 \mathrm{~L} / \mathrm{h}$, full scan acquisition mode, scan range 100-1500 (m/z), mass calibration: lockspray with 278.1141 Da and 556.2771 Da of leucine encephalin.

\section{6. Statistical analysis}

The concentrations of amatoxins and phallotoxins were expressed as mean \pm SD. To estimate the phylogenetic relationships of the ten Amanita species using cyclopeptide profiles, the nineteen main chromatographic peaks were manually scored as binary data for the presence or absence of the toxins, A table containing this binary information was used to calculate Jaccard's pairwise similarity coefficients using the SIMQUAL program (Similarity for Qualitative Data) of NTSYS-pc Version 2.1 (Rohlf, 2000). The phylogenetic tree was performed using the UPGMA (unweighted pair-group method with arithmetic averages) clustering method of NTSYS-pc Version 2.1.

\section{Results}




\subsection{Mushroom identification and phylogenetic analysis based on ITS sequences}

The species of Amanita were identified by morphological characteristics, and the identification were confirmed by the ITS sequences (Table 1). The resulting tree (Fig. 2) based on ITS sequences strongly resolved the taxa examined into seven lineages comprising nine phylogenetic species. These results are consistent with the results of phylogeny inferred from combined datasets (nrLSU, rpb2, efl- $\alpha$ and $\beta$-tubulin) and ITS sequences (Cai et al., 2014).

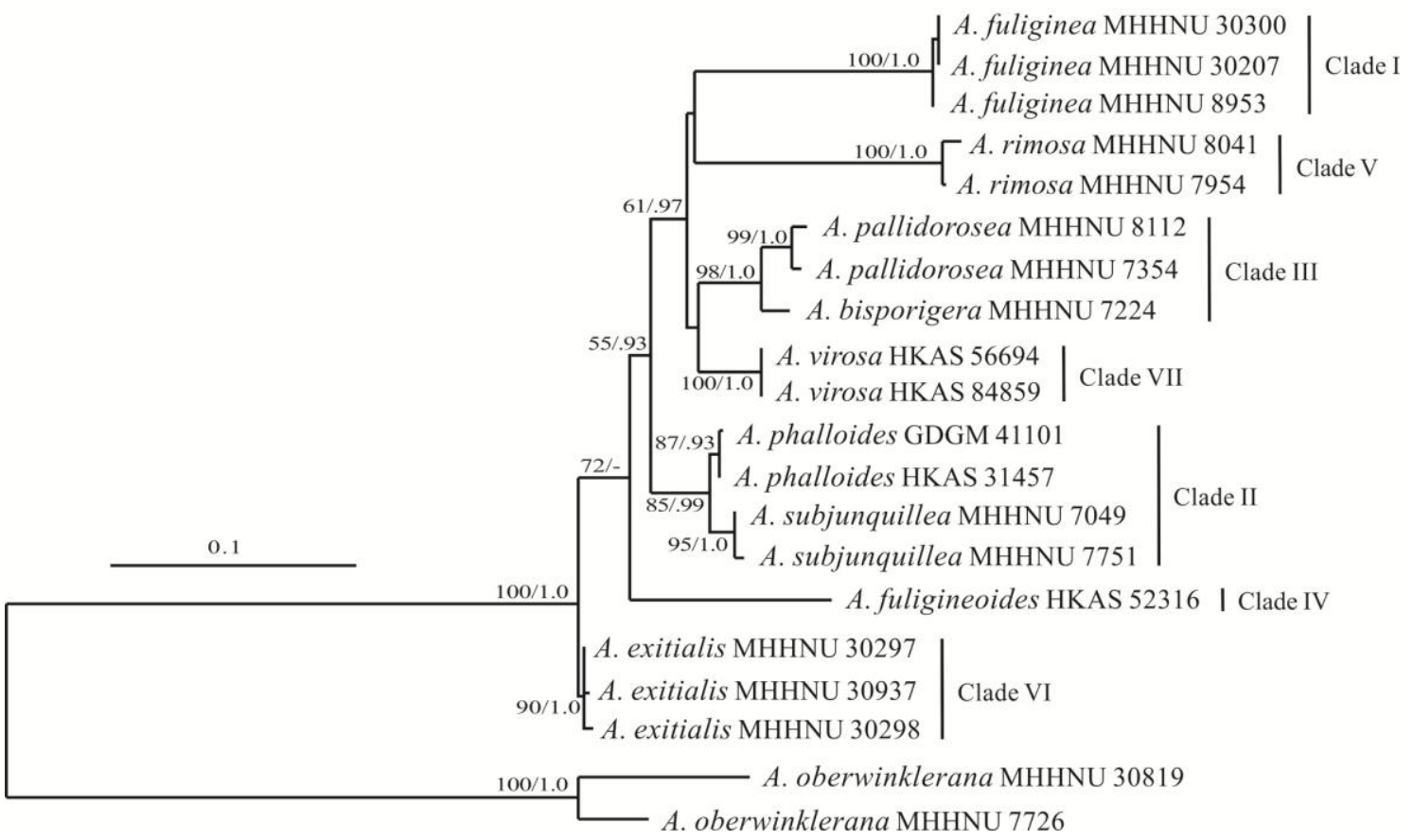

Fig. 2. Phylogenetic tree generated from maximum likelihood analysis based on ITS sequences.

Only maximum likelihood bootstraps and Bayesian posterior probabilities over $50 \%$ and 0.90 are reported on the branches.

\subsection{Concentrations and distribution of amatoxins and phallotoxins in lethal Amanita species}

The HPLC profiles of crude extractions of ten Amanita species and the mixture of five standard 
samples were shown in Fig. 3 and Fig. S1. They indicated that there is a great diversity in major peaks among species. All lethal Amanita species belonging to the section Phalloideae showed amatoxins and phallotoxins, and in contrast, A. oberwinklerana of the section Lepidella contained no amatoxins or phallotoxins.

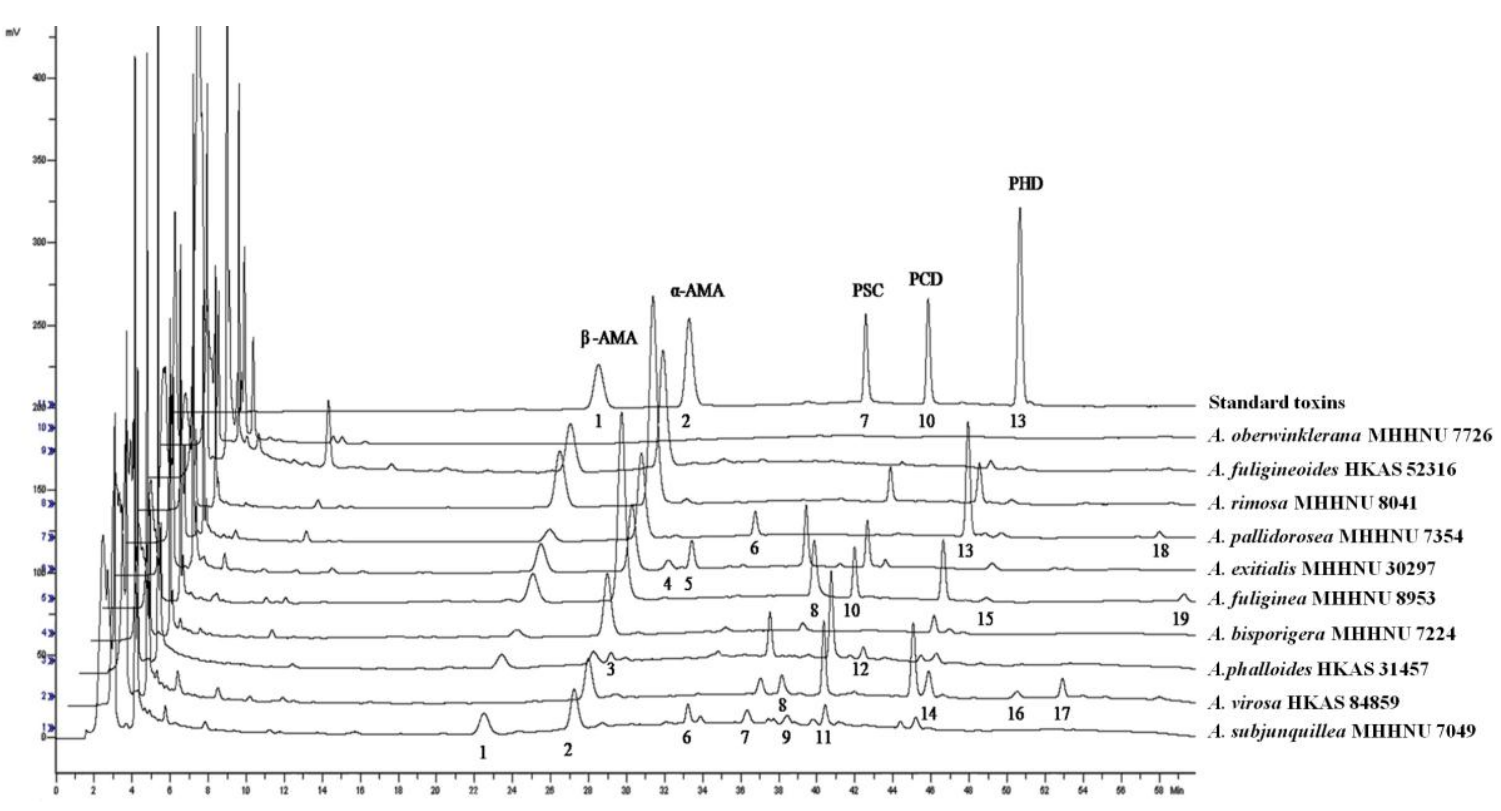

Fig. 3. HPLC profiles of cyclopeptide toxins extracted from lethal Amanita species.

Amanita oberwinklerana, supposed to be a lethal amanita, possesses no cyclopeptide toxins.

The compositions of amatoxins ( $\alpha$-amanitin and $\beta$-amanitin) and phallotoxins (PSC, PCD and PHD) of lethal Amanita species are given in Table 2. The amounts of total toxins ranged from 3.27 to14.19 $\mathrm{mg} \mathrm{g}^{-1}$ dry weight. A. rimosa had the highest toxin content, up to fourfold more than that in $A$. phalloides, A. virosa and A. bisporigera. The amatoxins, which are the only toxins responsible for the death of humans, varied significantly in the species tested. first, the A. rimosa, A. fuligineoides, A. fuliginea, A. pallidorosea and A. subjunquillea contained more than $5.00 \mathrm{mg} \mathrm{g}^{-1}$ dry weight amatoxins, then the A. exitialis and A. bisporigera contained amatoxins from 3.00 to $5.00 \mathrm{mg} \mathrm{g}^{-1}$ dry weight, and 
finally the A. phalloides and A. virosa contained only less than $2.00 \mathrm{mg} \mathrm{g}^{-1}$ dry weight.

Table 2. Compositions of amatoxins and phallotoxins in lethal Amanita species $\left(\mathrm{mg} \mathrm{g}^{-1}\right.$ dry weight)

\begin{tabular}{|c|c|c|c|c|c|c|c|c|c|}
\hline \multirow{2}{*}{$\begin{array}{c}\text { Scientific Name and } \\
\text { Species no. }\end{array}$} & \multicolumn{3}{|c|}{ Amatoxins (A) } & \multicolumn{4}{|c|}{ Phallotoxins (P) } & \multirow{2}{*}{$\begin{array}{l}\text { Total } \\
\text { contents }\end{array}$} & \multirow[b]{2}{*}{$\mathrm{P} / \mathrm{A}$} \\
\hline & $\alpha$-AMA & $\beta$-AMA & Total & PSC & PCD & PHD & Total & & \\
\hline A. bisporigera MHHNU7224 & $2.47 \pm 0.06$ & $0.56 \pm 0.04$ & 3.03 & trace & 0 & $0.45 \pm 0.03$ & 0.45 & 3.48 & 0.15 \\
\hline A. exitialis MHHNU30297 & $2.20 \pm 0.13$ & $2.16 \pm 0.20$ & 4.36 & $1.50 \pm 0.01$ & $1.39 \pm 0.01$ & 0 & 2.89 & 7.25 & 0.66 \\
\hline A. exitialis MHHNU30298 & $2.27 \pm 0.01$ & $2.16 \pm 0.14$ & 4.43 & $1.13 \pm 0.01$ & $0.77 \pm 0.01$ & 0 & 1.90 & 6.33 & 0.43 \\
\hline A. exitialis MHHNU 30937 & $1.07 \pm 0.06$ & $0.98 \pm 0.02$ & 2.05 & $0.55 \pm 0.03$ & $0.67 \pm 0.01$ & 0 & 1.22 & 3.27 & 0.60 \\
\hline A. fuliginea MHHNU 30300 & $5.14 \pm 0.19$ & $1.20 \pm 0.06$ & 6.34 & $0.22 \pm 0.01$ & $0.18 \pm 0.01$ & $1.19 \pm 0.10$ & 1.59 & 7.93 & 0.25 \\
\hline A. fuliginea MHHNU 30207 & $4.72 \pm 0.11$ & $1.31 \pm 0.04$ & 6.03 & $0.26 \pm 0.01$ & $0.14 \pm 0.01$ & $1.07 \pm 0.02$ & 1.47 & 7.50 & 0.24 \\
\hline A. fuliginea $\mathrm{MHHNU} 8953$ & $7.14 \pm 0.50$ & $1.89 \pm 0.12$ & 9.03 & $0.21 \pm 0.01$ & $1.40 \pm 0.09$ & $1.35 \pm 0.09$ & 2.96 & 11.99 & 0.33 \\
\hline A. fuligineoides HKAS 52316 & $4.72 \pm 0.21$ & $4.13 \pm 0.17$ & 8.85 & 0 & trace & $0.25 \pm 0.01$ & 0.25 & 9.10 & 0.03 \\
\hline A. pallidorosea MHHNU 8112 & $4.13 \pm 0.02$ & $1.27 \pm 0.02$ & 5.40 & 0 & trace & $2.87 \pm 0.09$ & 2.87 & 8.27 & 0.53 \\
\hline A. pallidorosea MHHNU 7354 & $4.09 \pm 0.16$ & $2.17 \pm 0.12$ & 6.26 & 0 & trace & $1.62 \pm 0.10$ & 1.62 & 7.88 & 0.26 \\
\hline A. phalloides HKAS 31457 & $0.50 \pm 0.02$ & $0.94 \pm 0.05$ & 1.44 & $1.30 \pm 0.02$ & $2.71 \pm 0.06$ & $0.26 \pm 0.01$ & 4.27 & 5.71 & 2.97 \\
\hline A. phalloides GDGM41101 & $0.85 \pm 0.07$ & $0.39 \pm 0.02$ & 1.24 & $0.79 \pm 0.06$ & $1.80 \pm 0.08$ & $0.22 \pm 0.02$ & 2.81 & 4.05 & 2.27 \\
\hline A. rimosa MHHNU 8041 & $8.08 \pm 0.37$ & $4.17 \pm 0.59$ & 12.25 & 0 & $1.05 \pm 0.03$ & $0.89 \pm 0.03$ & 1.94 & 14.19 & 0.16 \\
\hline A. rimosa MHHNU 7954 & $5.34 \pm 0.22$ & $3.44 \pm 0.12$ & 8.78 & 0 & $1.74 \pm 0.07$ & $0.72 \pm 0.02$ & 2.46 & 11.24 & 0.28 \\
\hline A. subjunquillea MHHNU 7049 & $1.69 \pm 0.01$ & $1.45 \pm 0.09$ & 3.14 & $0.60 \pm 0.01$ & trace & $0.25 \pm 0.01$ & 0.85 & 3.99 & 0.27 \\
\hline A. subjunquillea MHHNU 7751 & $5.27 \pm 0.01$ & $3.68 \pm 0.02$ & 8.95 & $0.33 \pm 0.08$ & $1.35 \pm 0.01$ & $2.04 \pm 0.08$ & 3.72 & 12.67 & 0.42 \\
\hline A. virosa HKAS 84859 & $1.60 \pm 0.09$ & 0 & 1.60 & $0.76 \pm 0.02$ & $2.09 \pm 0.01$ & $1.64 \pm 0.02$ & 4.49 & 6.09 & 2.81 \\
\hline A. virosa HKAS 56694 & $1.34 \pm 0.04$ & 0 & 1.34 & trace & $1.81 \pm 0.10$ & $0.99 \pm 0.04$ & 2.80 & 4.14 & 2.09 \\
\hline
\end{tabular}

The distribution of amatoxins and phallotoxins varied significantly in lethal amanitas, the amatoxin content was higher than phallotoxin content in A. bisporigera, A. exitialis, A. fuliginea, A. fuligineoides, 
A. fuligineoides, A. rimosa and A. subjunquillea, the ratios of which ranged from twofold to sevenfold, up to thirty-fivefold in the case of A. fuligineoides. Contrarily, phallotoxin content was higher than amatoxin content in A. phalloides and A. virosa, which contained twofold to threefold more phallotoxins than amatoxins (Table 2).

\subsection{Concentration and distribution of amatoxins and phallotoxins in different tissues}

Fully developed carpophores of four species (A. exitialis, A. fuliginea, A. pallidorosea and A. rimosa) were divided into three sections: pileus, stipe and volva. Each tissue underwent identical extraction and HPLC procedures, and the contents of amatoxins and phallotoxins were shown in Fig. 4.

The results indicated that, for all toxins tested, the highest concentrations were found in pileus followed by stipe, and the volva had the lowest amount of toxins.
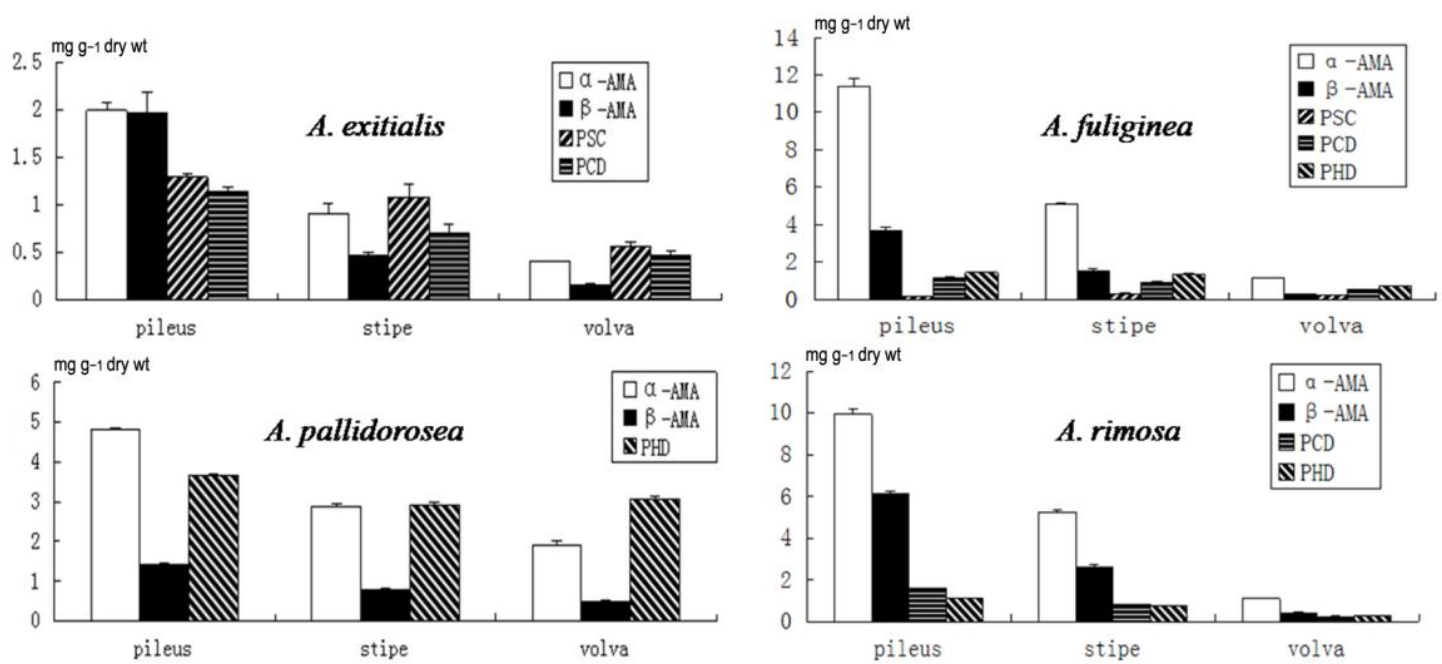

Fig. 4. The content and distribution of amatoxins and phallotoxins in different tissues of lethal Amanita species

Figure 4 also revealed that the distribution of amatoxins and phallotoxins varied in different tissues.

The total amount of amatoxins was higher than that of phallotoxins in pileus, but the ratio of 
phallotoxins to amatoxins in stipe and volva was higher than that in pileus, with the volva being the highest. In A. exitialis, and A. fuliginea, the amount of phallotoxins in the volva was higher than that of amatoxins.

Table 3. Masses and formulae of the main compounds

\begin{tabular}{|c|c|c|c|c|c|}
\hline Peak number & Compounds & True Mass (Da) & True formulae & Observed Masses(Da) & Observed formulae \\
\hline & & (Clarke et al., 2012) & (Clarke et al., 2012) & & \\
\hline 1 & $\beta$-amanitin & 919.3382 & $\mathrm{C}_{39} \mathrm{H}_{53} \mathrm{~N}_{9} \mathrm{O}_{15} \mathrm{~S}$ & $920.3458\left[\mathrm{M}+\mathrm{H}^{+}\right], 958.3026\left[\mathrm{M}+\mathrm{K}^{+}\right]$ & $\mathrm{C}_{39} \mathrm{H}_{53} \mathrm{~N}_{9} \mathrm{O}_{15} \mathrm{~S}$ \\
\hline 2 & $\alpha$-amanitin & 918.3542 & $\mathrm{C}_{39} \mathrm{H}_{54} \mathrm{~N}_{10} \mathrm{O}_{14} \mathrm{~S}$ & $919.3602\left[\mathrm{M}+\mathrm{H}^{+}\right], 957.3159\left[\mathrm{M}+\mathrm{K}^{+}\right]$ & $\mathrm{C}_{39} \mathrm{H}_{54} \mathrm{~N}_{10} \mathrm{O}_{14} \mathrm{~S}$ \\
\hline 5 & Unknown & - & - & $849.3058\left[\mathrm{M}+\mathrm{H}^{+}\right], 887.2672\left[\mathrm{M}+\mathrm{K}^{+}\right]$ & $\mathrm{C}_{31} \mathrm{H}_{48} \mathrm{~N}_{10} \mathrm{O}_{16} \mathrm{~S}$ \\
\hline 6 & Unknown & - & - & $819.2964\left[\mathrm{M}+\mathrm{H}^{+}\right], 857.2551\left[\mathrm{M}+\mathrm{K}^{+}\right]$ & $\mathrm{C}_{35} \mathrm{H}_{46} \mathrm{~N}_{8} \mathrm{O}_{13} \mathrm{~S}$ \\
\hline 7 & Phallisacin & 862.3167 & $\mathrm{C}_{37} \mathrm{H}_{50} \mathrm{~N}_{8} \mathrm{O}_{14} \mathrm{~S}$ & $863.3229\left[\mathrm{M}+\mathrm{H}^{+}\right], 901.2881\left[\mathrm{M}+\mathrm{K}^{+}\right]$ & $\mathrm{C}_{37} \mathrm{H}_{50} \mathrm{~N}_{8} \mathrm{O}_{14} \mathrm{~S}$ \\
\hline 8 & Amaninamide & 902.3593 & $\mathrm{C}_{39} \mathrm{H}_{54} \mathrm{~N}_{10} \mathrm{O}_{13} \mathrm{~S}$ & $903.3646\left[\mathrm{M}+\mathrm{H}^{+}\right], 941.3211\left[\mathrm{M}+\mathrm{K}^{+}\right]$ & $\mathrm{C}_{39} \mathrm{H}_{54} \mathrm{~N}_{10} \mathrm{O}_{13} \mathrm{~S}$ \\
\hline 10 & Phallacidin & 846.3218 & $\mathrm{C}_{37} \mathrm{H}_{50} \mathrm{~N}_{8} \mathrm{O}_{13} \mathrm{~S}$ & $847.3301\left[\mathrm{M}+\mathrm{H}^{+}\right], 885.2855\left[\mathrm{M}+\mathrm{K}^{+}\right]$ & $\mathrm{C}_{37} \mathrm{H}_{50} \mathrm{~N}_{8} \mathrm{O}_{13} \mathrm{~S}$ \\
\hline 13 & Phalloidin & 788.3163 & $\mathrm{C}_{35} \mathrm{H}_{48} \mathrm{~N}_{8} \mathrm{O}_{11} \mathrm{~S}$ & $789.3251\left[\mathrm{M}+\mathrm{H}^{+}\right], 827.2801\left[\mathrm{M}+\mathrm{K}^{+}\right]$ & $\mathrm{C}_{35} \mathrm{H}_{48} \mathrm{~N}_{8} \mathrm{O}_{11} \mathrm{~S}$ \\
\hline 14 & Phalloidin II & 788.3163 & $\mathrm{C}_{35} \mathrm{H}_{48} \mathrm{~N}_{8} \mathrm{O}_{11} \mathrm{~S}$ & $789.3215\left[\mathrm{M}+\mathrm{H}^{+}\right], 827.2786\left[\mathrm{M}+\mathrm{K}^{+}\right]$ & $\mathrm{C}_{35} \mathrm{H}_{48} \mathrm{~N}_{8} \mathrm{O}_{11} \mathrm{~S}$ \\
\hline 16 & Viroidin & 896.3586 & $\mathrm{C}_{38} \mathrm{H}_{56} \mathrm{~N}_{8} \mathrm{O}_{15} \mathrm{~S}$ & $897.3676\left[\mathrm{M}+\mathrm{H}^{+}\right], 935.3234\left[\mathrm{M}+\mathrm{K}^{+}\right]$ & $\mathrm{C}_{38} \mathrm{H}_{56} \mathrm{~N}_{8} \mathrm{O}_{15} \mathrm{~S}$ \\
\hline 17 & Unknown & - & - & $915.3806\left[\mathrm{M}+\mathrm{H}^{+}\right], 953.3282\left[\mathrm{M}+\mathrm{K}^{+}\right]$ & $\mathrm{C}_{38} \mathrm{H}_{59} \mathrm{~N}_{8} \mathrm{O}_{16} \mathrm{~S}$ \\
\hline
\end{tabular}

\subsection{Mass spectrometry analysis of the main compounds}

Nineteen compounds with clearly distinguishable peaks were identified and purified by multiple HPLC runs using the same column. Over $90 \%$ purity was achieved for every compound, and the samples were then analyzed with UPLC-QTOF MS/MS. The results showed that eleven out of nineteen compounds generated effective MS information, and eight compounds did not give strong signal due to lower sample concentrations. The eleven compounds were reported in Table 3 and Fig S2. Peaks 1, 2, 7 , 10 had the masses corresponding to that of $\beta$-amanitin, $\alpha$-amanitin, phallisacin, and phallacidin 
respectively. Peaks 13 and 14 had the same mass that corresponds to that of phalloidin (see Table 3).

Peak 13 had the same elution time of the standard phalloidin, we therefore concluded that peak 13 is phalloidin, while peak 14 is likely to be an analogue of phalloidin, which was named "phalloidin II" here. Peaks 8 and 16 were identified to be amaninamide and viroidin according to the masses of 902.3592 and 896.3585. Peaks 5, 6 and 17 generated accurate masses, but there were no corresponding known compounds.

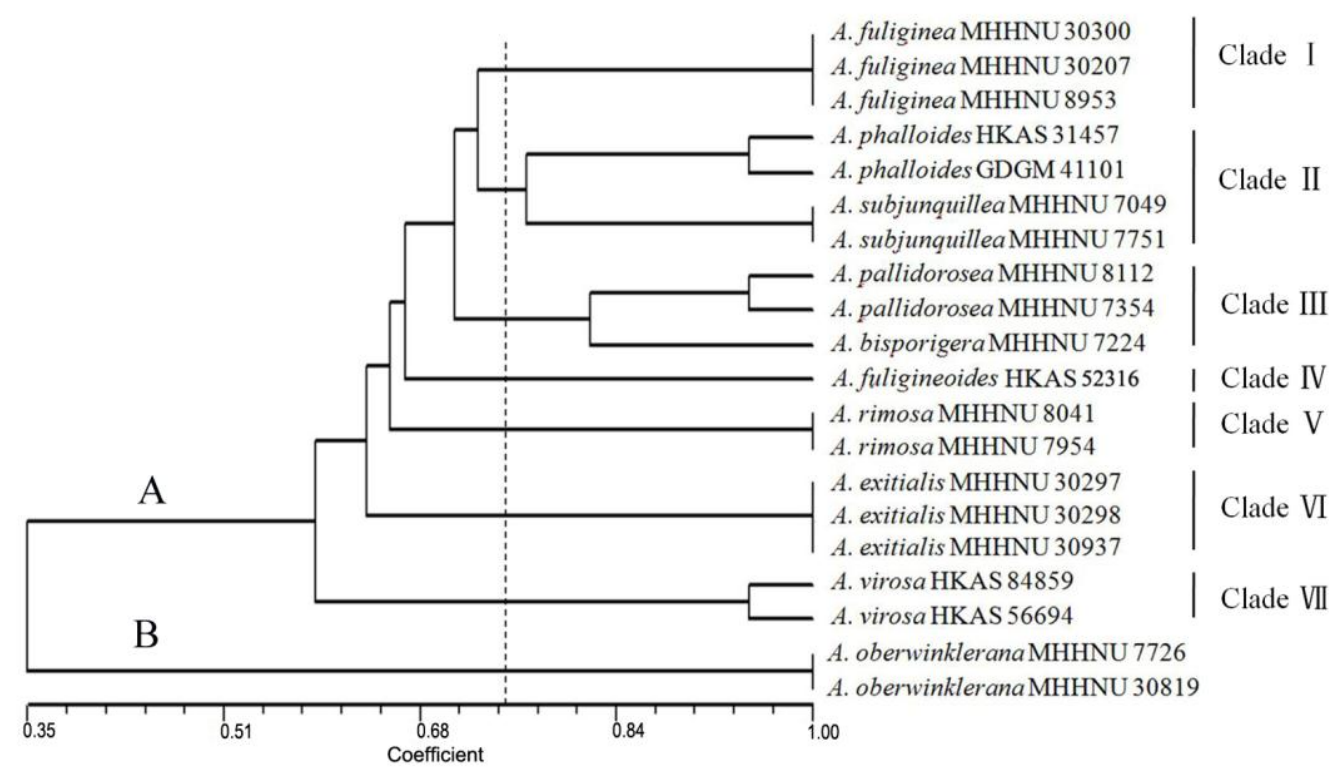

Fig. 5. Dendrogram of lethal Amanita sp. based on toxin profiles

\subsection{Phylogenetic relationships based on the cyclopeptide profiles}

A dendrogram generated from chromatographic profiles was shown in Fig. 5. The result of the analysis strongly supported two major monophyletic clades (A and B), which corresponded respectively to the lethal amanita group containing cyclopeptides in section Phalloideae and to members in section Lepidella that contain no cyclopeptides. Within clade A, seven lineages were supported based on the similarity coefficients level of 75\%: (1) the A. fuliginea lineage (Clade I ), (2) 
A. phalloides and A. subjunquillea lineage (Clade II), (3) A. pallidorosea and A. bisporigera lineage (Clade III), (4) A. fuligineoides lineage (Clade IV), (5) A. rimosa lineage (Clade V), (6) A. exitialis lineage (Clade VI), and (7) A. virosa lineage (Clade VII).

\section{Discussion}

\section{Concentration of amatoxins and phallotoxins between lethal Amanita species}

High performance liquid chromatography (HPLC) technology is the most widely favored method for the qualitative and quantitative analysis of amatoxins and phallotoxins in Amanita tissues and clinical serum and urine samples in the past 20 years (Enjalbert et al., 1992, 1993; Defendenti et al., 1998; Mcknight et al., 2010; $\mathrm{Hu}$ et al., 2012). The contents of amatoxins and phallotoxins of the Amanita species determined by HPLC are shown in Table 4. The amounts of $\alpha$-amanitin in $A$. phalloides, A. virosa and A. bisporigera collected from Europe and North America, were 0.67-2.95, 1.39 and $1.70 \mathrm{mg} \mathrm{g}^{-1}$ dry weight respectively (Enjalbert et al., 1993; Mcknight et al., 2010; Sgambelluri et al., 2014; Kaya et al., 2015; Garcia et al., 2015). In this study, we showed that the amounts of a-amanitin in A. phalloides, A. virosa and A. bisporigera, also collected from Europe and North America, were $0.50-0.85,1.34-1.60$ and $2.47 \mathrm{mg} \mathrm{g}^{-1}$ dry weight respectively. Also, our previous studies showed that the amounts of $\alpha$-amanitin in A. exitialis and A. fuliginea collected from China were 2.47-5.16 and $9.31 \mathrm{mg} \mathrm{g}^{-1}$ dry weight, respectively (Chen et al., 2003; Hu et al., 2012). In our present study, the amounts of $\alpha$-amanitin in A. exitialis and A. fuliginea ranged from 1.07 to 2.27 and 4.72 to $7.14 \mathrm{mg} \mathrm{g}^{-1}$ dry weight respectively. These results indicated that our present finding is in accord with the data in the literature, which lends support to the validity of our methodology. 
Table 4. Contents of amatoxins and phallotoxins in Amanita species relative to our present study determined by HPLC in literature ${ }^{\text {a }}$

\begin{tabular}{|c|c|c|c|c|c|c|c|}
\hline \multirow[t]{2}{*}{ Species } & \multirow[t]{2}{*}{ Tissue and origin } & \multicolumn{2}{|c|}{ Amatoxins } & \multicolumn{3}{|c|}{ Phallotoxins } & \multirow[t]{2}{*}{ references } \\
\hline & & $\alpha$-AMA & $\beta$-АMA & PSC & PCD & PHD & \\
\hline \multirow[t]{6}{*}{ A. phalloides } & Carpophores (France) ${ }^{b}$ & 1.54 & 1.41 & 1.31 & 2.32 & 1.32 & Enjalbert et al. 1993 \\
\hline & Carpophores (Turkey) ${ }^{\mathrm{c}}$ & $2.80 \pm 0.13$ & $2.38 \pm 0.06$ & $\underbrace{d}$ & $2.12 \pm 0.06$ & $1.32 \pm 0.01$ & Kaya et al. 2015 \\
\hline & Pileus (Turkey) ${ }^{\mathrm{c}}$ & $2.95 \pm 0.05$ & $2.53 \pm 0.03$ & & $2.27 \pm 0.02$ & $1.40 \pm 0.03$ & Kaya et al. 2015 \\
\hline & Pileus (Portugal) $^{\mathrm{c}}$ & $0.67-0.78$ & - & - & - & - & Garcia et al. 2015 \\
\hline & Carpophores (Italy) $^{\mathrm{c}}$ & 1.33 & - & - & - & - & Sgambelluri et al., 2014 \\
\hline & Carpophores (USA) $^{\mathrm{c}}$ & 0.88 & - & - & - & - & Sgambelluri et al., 2014 \\
\hline \multirow[t]{2}{*}{ A. virosa } & Carpophores (Italy) $^{\mathrm{c}}$ & 1.39 & 0 & - & - & - & Sgambelluri et al., 2014 \\
\hline & Pileus (Japan) ${ }^{\mathrm{b}}$ & 6.03 & 4.53 & & & 3.01 & Ahmed et al. 2010 \\
\hline A. bisporigera & Pileus (USA) $^{\mathrm{c}}$ & $1.70 \pm 0.68$ & - & - & $2.71 \pm 0.65$ & $11.98 \pm 1.66$ & Mcknight et al., 2010 \\
\hline \multirow[t]{2}{*}{ A. exitialis } & Pileus (China) ${ }^{\mathrm{c}}$ & $2.47 \pm 0.08$ & $2.44 \pm 0.07$ & - & - & - & Hu et al., 2012 \\
\hline & Pileus (China) ${ }^{\mathrm{c}}$ & 5.16 & 1.76 & - & 1.11 & 0 & Chen et al., 2003 \\
\hline A. fuliginea & Pileus (China) ${ }^{\mathrm{c}}$ & 9.31 & 1.04 & - & 0.71 & 1.46 & Chen et al., 2003 \\
\hline A. pallidorosea & Carpophores (China) ${ }^{\mathrm{c}}$ & 3.83 & - & - & - & - & Cao et al., 2011 \\
\hline A. subjunquillea & Carpophores (China) $^{\mathrm{c}}$ & 2.39 & 1.65 & - & - & 0.40 & Bao et al., 2005 \\
\hline
\end{tabular}

a All values are in $\mathrm{mg} \mathrm{g}^{-1}$ dry weight, assuming $1 \mathrm{~g}$ dry weight $=12.5 \mathrm{~g}$ fresh tissue,

b Fresh tissue, c Dry tissue, d "_-" means the toxin was not detected.

Table 2 and Fig. 2 showed that there was a diversity in compositions and contents of amatoxins and phallotoxins among the lethal Amanita species. To our knowledge, this is the first report of the analysis of amatoxins and phallotoxins in A. rimosa and A. fuligineoides. All lethal Amanita species tested contained $\alpha$-amanitn and $\beta$-amanitin except $A$. virosa. No $\beta$-amanitin was detected in $A$. virosa, which is consistent with the latest result by means of HPLC (Sgambelluri et al., 2014) and earlier result by means of thin-layer chromatograms (Yocum \& Simons, 1977). However, Ahmed et al. (2010) reported 
that the levels of $\beta$-amanitin was comparable to that of $\alpha$-amanitin in a specimen of $A$. virosa from Japan. We suppose that the species was likely identified inaccurately, as several Amanita species that possess white carpophores and are endemic to East Asia, are similar to A. virosa; therefore it is difficult to identify by morphologic characters alone.

A survey of the toxin composition of A. phalloides indicated that five components, $\alpha$-amanitin, $\beta$-amanitin, PCD, PHD and PSC, are the predominant components, representing $92 \%$ of the total toxin content (Enjalbert et al., 1993). Therefore we chose these five components for quantitative analysis in this study. Our results showed that the amounts of total toxins varied significantly in different lethal Amanita species, ranged from 3.27 to $14.19 \mathrm{mg} \mathrm{g}^{-1}$ dry weight. Because the responsible toxins for fatal human poisoning are amatoxins, not phallotoxins (Wieland, 1986; Karlson-Stiber \& Persson 2003), the evaluation of the amatoxin content of Amanita species is more important. The present results (Table 2) showed that the amounts of amatoxins in lethal Amanita species ranged from 1.24 to $12.25 \mathrm{mg} \mathrm{g}^{-1}$ dry weight. The amounts of amatoxins in East Asian Amanita species, such as A. fuliginea, A. rimosa, A. exitialis, A. fuligineoides, A. pallidorosea and A. subjunquillea are significantly higher than those in European and North America species, such as A. phalloides, A. virosa and A. bisporigera. The average content of amatoxins in East Asian Amanita species are about three to eight times the amount in $A$. phalloides, which was responsible for most of mortality in Europe. The data indicate that the East Asian Amanita species are more toxic than species from Europe and North America. In fact, all of these East Asian Amanita species except A. fuligineoides and A. subjunquillea were collected from the field near investigated mushroom poisoning accidents. In our investigation of mushroom poisoning cases in southern China from 1994 to 2012, we found that poisoning induced by the ingestion of homogeneous A. fuliginea or A. exitialis mushrooms could lead to 60-100\% of mortality (Chen et al., 2014). One case 
of mushroom poisoning caused by A. pallidorosea have been reported in China, the mortality rate was $43 \%$ in this case (Cao et al., 2011). Another incident caused by A. pallidorosea was investigated in Guizhou Province in 2014 by us, in which case 2 patients died with 100\% mortality. In 2015, we investigated two cases of mushroom poisoning caused by A. rimosa in Hunan and Jiansu provinces,

China, which involved 8 patients and 5 deaths, with $62.5 \%$ mortality.

\section{Distribution of amatoxins and phallotoxins in different lethal Amanita species and tissues}

The toxin composition in various carpophore tissues of Amanita phalloides, which was subdivided into six parts, namely, cap (pileus), gills, ring, stipe, volva and bulb, were comprehensively and systematically evaluated by Enjalbert et al. (1993, 1996, 1999). The results showed that variation of the toxin concentration in the different tissues was particularly large, and the content of phallotoxins was higher than the content of amatoxins in all tissues, the ratio of phallotoxins to amatoxins (P/A) for the various tissues indicated that bulb and volva contained four times more phallotoxins than amatoxins, whereas cap, gills, ring and stipe group had 1.5 times more phallotoxins than amatoxins. In addition, the environmental factors, mainly the soil type, clearly have an effect on the toxin composition in $A$. phalloides. Our present study indicated that content of phallotoxins was higher than that of amatoxins in A. phalloides and A. virosa: the result of $A$. phalloides $(\mathrm{P} / \mathrm{A}=2.27-2.97)$ is consistent with the report by Enjalbert et al. $(1993,1996,1999)$, and the result of $A$. virosa $(\mathrm{P} / \mathrm{A}=2.09-2.81)$ is in line with the report by Yocum \& Simons (1977). In contrast, the phallotoxin content was significantly lower than that of the amatoxins in all of other lethal Amanita species, and the ratio of phallotoxins to amatoxins (P/A) from 0.15 to 0.66 , the P/A being 0.03 in A. fuligineoides. That is to say that these Amanita species contained about twofold to sevenfold more amatoxins than phallotoxins, even thirty-fivefold in the case of A. fuligineoides. Interestingly, all of these lethal Amanita species except A. bisporigera are 
endemic to East Asia. Furthermore, the composition and distribution of amatoxins and phallotoxins in different tissues (pileus, stipe and volva) of A. exitialis, A. fuliginea, A. pallidorosea and A. rimosa were analyzed and the results are in agreement with the previous reports in A. phalloides (Enjalbert et al., 1993, 1996, 1999; Kaya et al., 2015), in A. phalloides var. alba (Kaya et al., 2013) and in A. exitialis (Hu et al., 2003; Li et al., 2014a).

\section{Cyclopeptides in lethal Amanita species}

Seven cyclopeptides were affirmatively identified in our present study, of which, $\beta$-amanitin, $\alpha$-amanitin, phallisacin, phallacidin, phalloidin and viroidin were identified by the both of elution time and hi-res mass. A phalloidin analogue ("phalloidin II") was identified according to the exact molecular weight and the formula. The analogues of phallotoxins were also reported in the previous literature (Clarke et al., 2012; Sgambelluri et al., 2014).

Peak 8 generated an $\left[\mathrm{M}+\mathrm{H}^{+}\right]$ion of $903.3646(\mathrm{~m} / \mathrm{z})$ and an $\left[\mathrm{M}+\mathrm{K}^{+}\right]$ion of $941.3211(\mathrm{~m} / \mathrm{z})$, which could correspond to either amaninamide or $\gamma$-amanitin, as amaninamide and $\gamma$-amanitin have the same mass of 902.3592. Amaninamide was firstly reported from A. virosa (Buku et al., 1980). In recent years, amaninamide was detected and identified using HPLC-MS/MS method in $A$. virosa, $L$. brunneoincarnata and A. exitialis by Sgambelluri et al.(2014) and Deng et al. (2011), respectively. The HPLC profile peak was relatively high, but the two compounds (amaninamide and $\gamma$-amanitin) were not detected in A. phalloides (Sgambelluri et al.,2014). Also, Clarke et al. (2012) reported that fourteen amatoxins and phallotoxins were identified in A. phalloides by means of LC-UV-ToF/MS system and $\gamma$-amanitin could be detected, but amaninamide did not exist. These findings indicated that amaninamide mainly exists in A. virosa and other Amanita species, and $\gamma$-amanitin exists in $A$. phalloides and other Amanita species. In our present study, the HPLC profiles showed that the 
relatively high peak 8 exists in A. virosa, A. fuliginea and A. bisporigera, and only trace of peak 8 exist in A. phalloides. Therefore, we can speculate the compound of peak 8 was amaninamide. Also, of the seven affirmative cyclopeptides, viroidin was detected only in A. virosa. Virotoxins, which are monocyclic peptides, appear to be restricted to A. virosa (Wieland, 1986).

Of the eleven identified compounds, three compounds (peaks 5, 6 and 17) generated accurate masses without corresponding to any reported Amanita cyclic peptide toxins. The peak 5 compound with higher concentration and a mass of 848 in A. exitialis was identified to be desoxoviroidin by Deng et al. (2011) and $\mathrm{Hu} \&$ Chen. (2014). In fact, the mass of desoxoviroidin is 880, so the identified desoxoviroidin in A. exitialis is incorrect. All twenty-two Amanita cyclopeptide toxins were discovered and isolated from A. phalloides and A. virosa by thin-layer chromatography (TLC) or high-performance TLC (HPTLC) before the 1980s, and no further peptide toxins have been discovered since then. Now, the more sensitive and effective HPLC methods have been developed, and new lethal Amanita species are being discovered continuously in recent years in East-Asia. Furthermore, the findings on the molecular diversity of toxin gene families in lethal Amanita mushrooms indicated nearly $33.7 \%$ of peptides sequences remain unknown ( $\mathrm{Li}$ et al., 2014b). These three factors provide the possibility for the discovery of new toxins. More than 10 milligrams of compound peak 5 have been prepared from A. exitialis in our laboratory; further studies on the structure and function are in progress.

\section{Phylogenetic relationships based on the cyclopeptides of lethal Amanita species}

The lethal amanitas are a group of cyclopeptide-containing mushrooms included in the genus Amanita sect. Phalloideae. The relationships of the lethal Amanita species based on the morphological and molecular phylogenetic data were elucidated by Zhang et al. (2010) and by Cai et al. (2014). 
Twenty eight phylogenetic species were divided into nine major clades based on the multi-locus DNA gene fragment analysis in combination with biochemical and morphological analyses (Cai et al., 2014).

Amanita oberwinklerana was originally described from Japan (Yang and Doi 1999), which was also collected and reported from China (Yang and Li 2001; Yang, 2005, 2015). According to its morphological characteristics, this species was placed in section Phalloideae (Yang and Doi 1999; Yang and Li 2001; Yang 2005). However, the results of molecular analyses from both ITS and combined dataset (nrLSU, rpb2, ef1- $\alpha$ and $\beta$-tubulin) showed that it appeared to be a member of section Lepidella (Zhang et al. 2010; Cai et al. 2014). In our present study, neither amatoxins nor phallotoxins were detected in A. oberwinklerana, this biochemical evidence strongly supported that $A$. oberwinklerana should be removed from the section Phalloideae and be allocated to the section Lepidella. Furthermore, two poisoning case caused by A. oberwinklerana were reported by us, which caused acute renal failure (Fu et al., in press), which is in accord with that the mushrooms in the Lepidella sections produce primarily acute renal injury (Graeme 2014; Kirchmair et al. 2012).

Vargas et al. (2011) determined the amatoxin and phallotoxin composition of twelve Amanita species in Colombia. Eleven of the twelve Amanita species analyzed in their report belong to section Amanita, Validae or Vaginatae, and only one species (A. bisporigera) belongs to section Phalloideae. However, their analysis showed that all of the analyzed species have, surprisingly, $\alpha$-amanitin in concentrations ranging from $50 \mathrm{ppm}$ to $6000 \mathrm{ppm}$. Based on our data and other previous literatures, we suppose that the results were inaccurate, because neither amatoxins nor phallotoxins have been detected in Amanita species of sections Amanita, Validae, Vaginatae and Lepidella (Hallen et al., 2002; Li and Oberlies, 2005; Cai et al., 2014 ). Furthermore, Hallen et al. (2007) demonstrated that the toxic genes (AMA1 and PHA1) were absent in the Amanita species outside of section Phalloideae. Also, the 
chosen 18 most informative chromatographic peaks for the dendrogram were located in zone between 0 and $5 \mathrm{~min}$, most of the peaks were not amatoxins and phallotoxins. Therefore, the results of the dendrogram in Vargas et al. (2011) could not reflect the relationship between toxic compounds and the taxonomy of the species.

In our present study, we generated a dendrogram by UPGMA method based on the cyclopeptide chromatographic profiles for the first time. The result of the analysis strongly supported that the nine analyzed Amanita species in section Phalloideae can be divided into seven lineages based on the similarity coefficients level of $75 \%$. Among them, A. phalloides and A. subjunquillea formed a clade, $A$. pallidorosea and A. bisporigera formed another clade. Interestingly, these results are remarkably consistent with the results of phylogenetic analysis based on their ITS sequences and the combined dataset (nrLSU, rpb2, efl- $\alpha$ and $\beta$-tubulin) (Cai et al., 2014). The data and prior reports raise the following questions, why is the dendrogram based on the cyclopeptide chromatographic profiles so similar to that based on the DNA sequences? What are the genetic bases of cyclopeptide diversity? And are there any unknown toxins present? Recent studies have demonstrated that the cyclopeptide toxins of Amanita mushrooms were synthesized on ribosomes and not by nonribosomal peptide synthetases (NRPSs). The genes encoding the amatoxins and phallotoxins belong to the MSDIN genes family, characterized by highly conserved amino acid sequences flanking a hypervariable toxin region that gives rise to the linear peptides corresponding to the mature toxins (Hallen et al., 2007; Luo et al., 2009; 2010). Analysis of the MSDIN family members identified that there were significant diversities and consensus in the toxin genes among the cyclopeptide-containing species of genus Amanita and Galerina and clarified that nearly $33.7 \%$ of the predicted peptide sequences are for unknown/uncharacterized products (Hallen et al., 2007; Luo et al., 2012; Li et al., 2014b). Also, the 
phylogenetic analysis of toxin gene $(a-A M A)$ demonstrated that the conservative phylogenetic relationships existed in the same genus, and significant differences were shown the genus level (Li et al., 2014b). Therefore, these results can well explain the diversity of cyclopeptides and the similarity between the dendrogram based on the cyclopeptides and those based on DNA sequences. Also, the unknown sequences of peptides indicated the existence of new toxins.

\section{Conflict of interest}

The authors declare that they have no conflict of interest.

\section{Authors' contributions}

Chen ZH conceived and designed the experiments; Tang SS, Zhou Q and Luo T detected and analyzed the cyclopeptide toxins, He ZM carried out the ITS DNA sequences of all Amanita species, Zhang P, Yang ZL and Cai Qing provided some Amanita materials and identified the species; Chen J performed the LC-MS/MS analysis and Chen ZH wrote the paper. All of the authors approved the final submission. All authors read and approved the final manuscript.

\section{Acknowledgments}

The authors are very grateful to Dr. Li TH and Deng WQ (Guangdong Institute of Microbiology, China) for providing Amanita phalloides sample collected from Italy for this study. Prof. Dr. Xie JW (Institute of Pharmacology and Toxicology, Academy of Military Medical Sciences, China) is acknowledged for providing the UPLC-QTOF MS/MS experiment guide. We also thank Dr. Luo H (Kunming Institute of Botany, Chinese Academy of Sciences, China) for the critically reviewing the manuscript. This study was supported by the National Science Foundation of China (Grants No. 31372118 and 30972073), the Research Foundation of Education Bureau of Hunan Province, China 
(Grant No. 13A059) and by the Joint Funds of the National Natural Science Foundation of China and Yunnan Provincial Government (Grant No. U1302263).

\section{Supplementary data}

Table S1. Data of presence or absence of the nine informative chromatographic peaks in Amanita species

Figure S1. HPLC proviles of standard toxins and extraction of ten Amanita species

Figure S2. QTOF-MS/MS information of the main compounds

\section{References}

Ahmed, W.H.A., Gonmori, K., Suzuki, M., Watanabe, K., Suzuki, O., 2010. Simultaneous analysis of $\alpha$-amanitin, $\beta$-amanitin, and phalloidin in toxic mushrooms by liquid chromatography coupled to time-of-flight mass spectrometry. Forensic Toxicol. 28, 69-76. doi: 10.1007/s11419-009-0089-7.

Bao, H., Bau, T., Li, Y., 2005. HPLC analysis of peptide toxins in seven species of Amanita. Junwu Yanjiu 3, 13-16. (in Chinese)

Benjamin, D.R., 1992. Mushroom poisoning in infants and children: The Amanita pantherina/muscaria group. J. Toxicol. Clin. Toxicol. 30, 13-22.

Berger, K.J., Guss, D.A., 2005. Mycotoxins revisited: Part I. J. Emerg. Med. 28, 53-62.

Buku, A.,Wieland, T., Bodenmüller, H., Faulstich, H., 1980. Amaninamide, a new toxin of Amanita virosa mushrooms. Experientia 36, 33-4.

Cai, Q., Tulloss, R.E., Tang, L.P., Tolgor, B., Zhang, P., Chen, Z.H., Yang, Z.L., 2014. Multi-locus phylogeny of lethal amanitas: implications for species diversity and historical biogeography. BMC 
Evol. Biol. 14,143. doi: 10.1186/1471-2148-14-143.

Cao, R.M., Liu, Z., Zhang, J., Liu, S.Q., Sun, Y.B., Zhang, J., Liu, S.H., Liu, L.Z., Cao, X.L., Jia, T.H., 2011. Disposal report of a case of mushroom poisoning caused by Amanita pallidorosea. J Shangdong Univ. Health Sci. 49, 160-164. (in Chinese).

Chen, Z.H., Hu, J.S., Zhang, Z.G., Zhang, P., Li, D.P., 2003. Determination and analysis of the main amatoxins and phallotoxins in 28 species of Amanita from China. Mycosystema 22, 565-573. (in Chinese)

Chen, Z.H., Zhang, P., Zhang, Z.G., 2014. Investigation and analysis of 102 mushroom poisoning cases in Southern China from 1994 to 2012. Fungal Divers. 64, 123-131. doi: $10.1007 / \mathrm{s} 13225-013-0260-7$.

Clarke, D.B., Lloyd, A.S., Robb, P., 2012. Application of liquid chromatography coupled to time-of-flight mass spectrometry separation for rapid assessment of toxins in Amanita mushrooms. Anal. Meth. 4, 1298-1309. doi: 10.1039/C2AY05575A.

Defendenti, C., Bonacina, E., Mauroni, M., Gelosa, L., 1998. Validation of a high performance liquid chromatographic method for alpha amanitin determination in urine. Forensic Sci. Int. 92, 59-68.

Deng, W.Q., Li, T.H., Xi, P.G., Gan, L.X., Xiao, Z.D., Jiang, Z.D., 2011. Peptide toxin components of Amanita exitialis basidiocarps. Mycologia 103, 946-949. doi: 10.3852/10-319.

Diaz, J.H., 2005. Syndromic diagnosis and management of confirmed mushroom poisonings. Crit. Care Med. 33, 427-36.

Enjalbert, F., Cassanas, G., Guinchard, C., Chaumont, J.P., 1996. Toxin composition of Amanita phalloides tissues in relation to the collection site, Mycologia 88, 909-921.

Enjalbert, F., Gallion, C., Jehl, F., Mohteil, H., Faulstich, H., 1993. Amatoxins and phallotoxins in 
Amanita species: high-performance liquid chromatographic determination. Mycologia 85, 579-584.

Enjalbert, F., Cassanas, G., Salhi, S.L., Guinchard, C., Chaumont, J.P., 1999. Distribution of the amatoxins and phallotoxins in Amanita phalloides. Influence of the tissues and the collection site. C. R. Acad. III 322, 855-862.

Enjalbert, F., Gallion, C., Jehl, F., Monteil, H., 1993. Toxin content, phallotoxin and amatoxin composition of Amanita phalloides tissues. Toxicon, 803-807.

Enjalbert, F., Rapior, S., Nouguier-Soulé, J., Guillon, S., Amouroux, N., Cabot, C., 2002. Treatment of amatoxin poisoning: 20-year retrospective analysis. J. Toxicol. Clin. Toxicol. 40, 715-57.

Enjalbert, F., Gallion, C., Jehl, F., Monteil, H., Faulstich, H., 1992. Simultaneous assay for amatoxins and phallotoxins in Amanita phalloides Fr. by high-performance liquid chromatography. J. Chromatogr. 598,227-236.

Faulstich, H., Buku, A., Bodenmuller, H., Dabrowski, J., Wieland, T., 1980. Virotoxins: Actin binding cycle peptides of Amanita virosa mushrooms. Biochemistry 19, 3334-3343.

Felsenstein, J., 1981. Evolutionary trees from DNA sequences: a maximum likelihood approach. J. Mol. Evol. 17, 368-376.

Fu, X. Y., Fu, B., He, Z. M., Gong, M. M., Li, Z. G., Chen, Z. H., 2016. Acute renal failure caused by Amanita oberwinklerana poisoning. Mycoscience (in press).

Garcia, J., Oliveira, A., de Pinho, P.G., Freitas, V., Carvalho, A., Baptista, P., Pereira, E., de Lourdes Bastos, M., Carvalho, F., 2015. Determination of amatoxins and phallotoxins in Amanita phalloides mushrooms from northeastern Portugal by HPLC-DAD-MS. Mycologia 107, 679-87. doi: $10.3852 / 14-253$. 
Graeme, K. A., 2014. Mycetism: A review of the recent literature. J. Med. Toxicol. 10: 173-189; doi: $10.1007 / \mathrm{s} 13181-013-0355-2$.

Hallen, H.E., Adams, G.C., Eicker, A., 2002. Amatoxins and phallotoxins in indigenous and introduced South African Amanita species. S. Afr. J. Bot. 68:322-326.

Hallen, H.E., Luo, H., Scott-Craig, J.S., Walton, J.D., 2007. Gene family encoding the major toxins of lethal Amanita mushrooms. Proc. Natl. Acad. Sci. U. S. A. 104, 19097-101.

Hongo, T., 1953. Large fungi of the Provinces of Omi and Yamashiro (4). J. Jpn. Bot. 28, 69-75.

Hu, J.S. Chen, Z.H., 2014. Isolation and preparation of cyclopeptide toxins by macroporous adsorptive resins combined with sephadex LH20 column chromatography from Amanita exitialis. Mycosystema 33, 549-559. (in Chinese).

Hu, J.S., Chen, Z.H., Zhang, Z.G., Zhang, P., 2003. Analysis of the main amatoxins and phallotoxins in Amanita exitialis - a new species in China. Acta Microbiol. Sin. 43, 642-646.

Hu, J.S., Zhang, P., Zeng, J., Chen, Z.H., 2012. Determination of amatoxins in different tissues and development stages of Amanita exitialis. J. Sci. Food Agric. 92, 2664-2667. doi: $10.1002 /$ jsfa.5685.

Imai, S., 1933. Studies on the Agaricaceae of Japan I. Volvate agarics in Hokkaido. Bot. Mag. Tokyo $47,423-432$.

Imazeki, R., Otani, Y., Hongo, T., 1988. Fungi of Japan. Yama-Kei Publisher, Tokyo.

Kaplan, C.D., Larsson, K.M., Kornberg, R.D., 2008. The RNA polymerase II trigger loop functions in substrate selection and is directly targeted by alpha-amanitin. Mol. Cell 30, 547-56. doi: 10.1016/j.molcel.2008.04.023.

Karlson-Stiber, C., Persson, H., 2003. Cytotoxic fungi-an overview. Toxicon 42: 339-349. 
Kaya, E., Karahan, S., Bayram, R., Yaykasli, K.O., Colakoglu, S., Saritas, A., 2015. Amatoxin and phallotoxin concentration in Amanita phalloides spores and tissues. Toxicol. Ind. Health 31, 1172-7. doi: 10.1177/0748233713491809.

Kaya, E., Yilmaz, I., Sinirlioglu, Z.A., Karahan, S., Bayram, R., Yaykasli, K.O., Colakoglu, S., Saritas, A., Severoglu, Z., 2013. Amanitin and phallotoxin concentration in Amanita phalloides var. alba mushroom. Toxicon 76, 225-33. doi: 10.1016/j.toxicon.2013.10.008.

Kim, K.H., Choi, S.U., Park, K.M., Seok, S.J., Lee, K.R., 2008. Cytotoxic constituents of Amanita subjunquillea. Arch. Pharm. Res. 31, 579-586. doi: 10.1007/s12272-001-1196-3.

Kirchmair, M., Carrilho, P., Pfab, R., Haberl, B., Felgueiras, J., Carvalho, F., Cardoso, J., Melo, I., Vinhas, J., Neuhauser, S., 2012. Amanita poisonings resulting in acute, reversible renal failure: new cases, new toxic Amanita mushrooms. Nephrol. Dial. Transplant. 27, 1380-1386. doi: $10.1093 /$ ndt/gfr511.

Li, C., Oberlies, N.H., 2005. The most widely recognized mushroom: chemistry of the genus Amanita. Life Sci. 78, 532-538.

Li, H.J., Xie, J.W., Zhang, S., Zhou, Y.J., Ma, P.B., Zhou, J., Sun, C.Y., 2015. Amanita subpallidorosea, a new lethal fungus from China. Mycol. Progress 14:43. doi: $10.1007 / \mathrm{s} 11557-015-1055-\mathrm{x}$.

Luo, H., Hallen-Adams, H.E., Scott-Craig, J.S., Walton, J.D., 2010. Colocalization of amanitin and a candidate toxin-processing prolyl oligopeptidase in Amanita basidiocarps. Eukaryot. Cell 9, 1891-900. doi: 10.1128/EC.00161-10.

Luo, H., Hallen-Adams, H.E., Scott-Craig, J.S., Walton J.D., 2012. Ribosomal biosynthesis of $\alpha$-amanitin in Galerina marginata. Fungal Genet. and Biol. 49, 123-129. doi: 
10.1016/j.fgb.2011.12.005.

Luo, H., Hallen-Adams, H.E., Walton, J.D., 2009. Processing of the phalloidin proprotein by prolyl oligopeptidase from the mushroom Conocybe albipes. J. Biol. Chem. 284, 18070-18077. doi: 10.1074/jbc.M109.006460.

Luo, H., Hong, S.Y., Sgambelluri, R.M., Angelos, E., Li, X., Walton, J.D., 2014. Peptide macrocyclization catalyzed by a prolyl oligopeptidase involved in $\alpha$-amanitin biosynthesis. Chem.

Biol. 21, 1610-1617. doi: 10.1016/j.chembiol.2014.10.015.

Li, P., Deng, W.Q., Li, T.H., 2014a. Molecular cloning of $\alpha$-amanitin and characterization of its expression pattern in different parts and development stages of Amanita exitialis fruitbody. Mycol. Progress 13, 1011-1016. doi: 10.1007/s11557-014-0988-9.

Li, P., Deng, W.Q., Li, T.H., 2014b. The molecular diversity of toxin gene families in lethal Amanita mushrooms. Toxicon 83, 59-68. doi: 10.1016/j.toxicon.2014.02.020.

Li, P., Deng, W.Q., Li, T.H., Song, B., Shen, Y.H., 2013. Illumina-based de novo transcriptome sequencing and analysis of Amanita exitialis basidiocarps. Gene 532, 63-71. doi: 10.1016/j.gene.2013.09.014.

Mao, X. L., 2006. Poisonous mushrooms and their toxins in China. Mycosystema 25, 345-363.

Mcknight, T.A., Mcknight, K.B., Skeels, M.C., 2010. Amatoxin and phallotoxin concentration in Amanita bisporigera spores. Mycologia 102, 763-765.

Michelot, D., Melendez-Howell, L. M., 2003. Amanita muscaria: chemistry, biology, toxicology, and ethnomycology. Mycol. Res. 107, 131-146.

Nguyen, V.T., Giannoni, F., Dubois, M.F., Seo, S.J., Vigneron, M., Kédinger, C., Bensaude, O., 1996. In vivo degradation of RNA polymerase II largest subunit triggered by alpha-amanitin. Nucleic 
Acids Res. 24, 2924-2929.

Rohlf, F.J., 2000. NTSYS-pc: Numerical Taxonomy and Multivariate Analysis System,Version 2.1.

New York: Exeter Software.

Ronquist, F., Huelsenbeck, J.P., 2003. MrBayes 3: Bayesian phylogenetic inference under mixed models. Bioinformatics, 19,1572-1574

Saviuc, P., Danel, V., 2006. New syndromes in mushroom poisoning. Toxicol. Rev. 25, 199-209.

Sgambelluri, R.M., Epis, S., Sassera, D., Luo, H., Angelos, E.R., Walton, J.D., 2014. Profiling of amatoxins and phallotoxins in the genus Lepiota by liquid Chromatography combined with UV absorbance and mass spectrometry. Toxins (Basel) 6, 2336-2347. doi: 10.3390/toxins6082336.

Vargas, N., Bernal, A., Sarria, V., Franco-Molano, A., Restrepo, S., 2011. Amatoxin and phallotoxin composition in species of the genus Amanita in Colombia: a taxonomic perspective. Toxicon 58, 583-590. doi: 10.1016/j.toxicon.2011.09.005.

Wang, D., Bushnell, D.A., Westover, K.D., Kaplan, C.D., Kornberg, R.D., 2006. Structural basis of transcription: role of the trigger loop in substrate specificity and catalysis. Cell 127, 941-954.

Wang, Y.L., Bao, H.Y., Xu, L., Tolgor, B., 2011. Determination of main peptide toxins from Amanita pallidorosea with HPLC and their antifungal action on Blastomyces albicans. Wei Sheng Wu Xue Bao 51, 1205-1211. (in Chinese)

Yang, Z. L., 2000. Species diversity of the genus Amanita (Basidiomycetes) in China. Acta Botanica Yunnanica 22, 135-142.

Yang, Z. L., 1997. Die Amanita-Arten von Südwestchina. Bibliotheca Mycologica 170, 1-240.

Yang, Z. L., 2005. Flora Fungorum Sinicorum Vol. 27, Amanitaceae. Science Press, Beijing. 1-258.

Yang, Z. L., 2015. Atlas of the Chinese species of Amanitaceae. Science Press, Beijing. 1-213. (in 


\section{Chinese)}

Yang, Z. L., Li, T. H., 2001. Notes on white Amanita of section Phalloideae (Amanitaceae) from China. Mycotaxon 78, 439-448.

Yang, Z.L., Cai, Q., Cui, Y.Y., 2016. Phylogeny, diversity and morphological evolution of Amanitaceae. Biosystematics and Ecology Series (in press).

Yang, Z.L., Doi, Y.,1999. A contribution to the knowledge of Amanita (Amanitaceae, Agaricales) in Bulletin of the National Science Museum. Series B, Botany 25, 107-130.

Yocum, R.R., Simons, D.M., 1977. Amatoxins and phallotoxins in Amanita species of the northeastern United States. Lloydia 40, 178-190.

Zhang, P., Chen, Z.H., Xiao, B., Tolgor, B., Bao, H.Y., Yang, Z.L., 2010. Lethal amanitas of East Asia characterized by morphological and molecular data. Fungal Divers. 42, 119-133. doi: $10.1007 / \mathrm{s} 13225-010-0018-4$. 